Check for updates

Cite this: Nanoscale Adv., 2019, 1, 4016

\title{
Insight into the dynamics of APOBEC3G protein in complexes with DNA assessed by high speed AFM $\dagger$
}

\author{
Yangang Pan, Luda S. Shlyakhtenko* and Yuri L. Lyubchenko (D)*
}

APOBEC3G (A3G) is a single-stranded DNA (sSDNA) binding protein that restricts the HIV virus by deamination of $d C$ to $d U$ during reverse transcription of the viral genome. $A 3 G$ has two zinc-binding domains: the N-terminal domain (NTD), which efficiently binds SSDNA, and the C-terminal catalytic domain (CTD), which supports deaminase activity of A3G. Until now, structural information on A3G has been lacking, preventing elucidation of the molecular mechanisms underlying its interaction with ssDNA and deaminase activity. We have recently built a computational model for the full-length A3G monomer and validated its structure using data obtained by time-lapse High-Speed Atomic Force Microscopy (HS AFM). Here time-lapse HS AFM is applied to directly visualize the structure and dynamics of A3G in complexes with ssDNA. Our results demonstrate a highly dynamic structure of A3G, where two domains of the protein fluctuate between compact globular and extended dumbbell structures. Quantitative analysis of our data revealed a substantial increase in the number of A3G dumbbell structures in the presence of the DNA substrate, suggesting that the interaction of A3G with the ssDNA substrate stabilizes this dumbbell structure. Based on these data, we proposed a model explaining the interaction of globular and dumbbell structures of A3G with ssDNA and suggested a possible role of the dumbbell structure in $A 3 G$ function.

Received 24th July 2019

Accepted 3rd September 2019

DOI: 10.1039/c9na00457b

rsc.li/nanoscale-advances individual domains, we recently ${ }^{28}$ built a computer model for full-length monomeric A3G. The model revealed the dynamics of A3G when two domains change their relative orientation and the protein transforms from a compact globular structure into an extended dumbbell structure. This model was validated by time-lapse high-speed AFM (HS-AFM), which enabled the direct observation of the transition between the globular and dumbbell structures of A3G. Importantly, the ratio between the two structures of A3G obtained from these experiments coincided with that obtained from simulations, which provides additional validation for the simulated model of the monomeric, fulllength structure and dynamics of A3G.

Here the HS-AFM methodology ${ }^{29-32}$ is utilized to visualize the dynamics of monomeric A3G in complex with ssDNA. To unambiguously identify the A3G-DNA complexes, a hybrid-DNA approach $^{17,28,33,34}$ was employed, and different types of DNA substrates were used to reveal the intramolecular dynamics of A3G. It was demonstrated that A3G forms complexes with ssDNA either in compact globular and/or dumbbell structures, but the population of the dumbbell structures of A3G considerably increased compared to that of the free protein. A clear dependence was also found for the yield of the dumbbell structures on the length of the ssDNA substrate. Interestingly, the number of dumbbell structures increases coincidently with the length of the ssDNA substrate. The use of different ssDNA substrates allowed us to observe one of the domains being transiently dissociated from ssDNA, demonstrating a very
Department of Pharmaceutical Sciences, College of Pharmacy, WSH, University of Nebraska Medical Center, Omaha, Nebraska 68198-6025, USA. E-mail: ylyubchenko@unmc.edu; lshlyakhtenko@unmc.edu

$\dagger$ Electronic supplementary information (ESI) available. See DOI: 10.1039/c9na00457b 
dynamic behavior of $\mathrm{A} 3 \mathrm{G}$ in the presence of the SSDNA substrate. Based on these results, we suggested a model to explain the role of the dynamics of $\mathrm{A} 3 \mathrm{G}$ in the interaction with ssDNA and form a hypothesis for its role in protein function.

\section{Results}

\section{Use of DNA substrates in high-speed AFM studies}

To examine the structure and dynamics of A3G in complexes with ssDNA, a hybrid-DNA approach was utilized, where ssDNA segments were fused with the DNA duplex, and HS-AFM was applied for unambiguous identification of the A3G ssDNA complexes. ${ }^{17,28,33,34}$ A3G complexes with three different hybrid DNA substrates, as used in this study, are illustrated in Fig. S1A$\mathrm{C} \uparrow$ and insets (i) and (ii) illustrate AFM images for $69 \mathrm{nt}$ tail and 69 nt gap hybrid DNA, respectively. A3G complexes with $69 \mathrm{nt}$ tail ssDNA (A) and $25 \mathrm{nt}$ tail SsDNA (B) show A3G bound to the ssDNA portion next to the dsDNA tag. The A3G complex with 69 nt gap ssDNA (C) shows the protein positioned in the ssDNA portion located between DNA duplexes. After assembly of A3G complexes, as described in the Materials and methods section, an aliquot was deposited on an APS mica surface for 2 minutes to allow complexes to bind to the surface, followed by rinsing of non-bound complexes and imaging without drying. After the A3G ssDNA complex of interest was selected on the AFM image, continuous frame-by-frame imaging of this complex was performed until A3G dissociated from the ssDNA substrate. The collected frames were then assembled into movies. The corresponding subsections below present the results of data analysis for the three different ssDNA substrates in complex with A3G.

\section{A3G in complex with the 69 nt tail ssDNA substrate}

Fig. 1 demonstrates the dynamics of A3G in complex with the 69 tail ssDNA substrate, where a few frames were selected from Movie $1 . \dagger$ The selected frames demonstrate a highly dynamic behavior of A3G in complex with the 69 nt tail ssDNA substrates, showing both globular and dumbbell structures of A3G. Frame 18 shows the globular conformation of A3G complexed with the 69 nt tail ssDNA. Frame 44 illustrates the transition of A3G from the globular to the dumbbell structure, in which both domains of A3G clearly separate from each other. Frames 56, 57, and 99 demonstrate the fluctuations in the distance between the two domains in the dumbbell structure of A3G, with the largest distance shown in frame 99. Later, the domains returned to the globular structure, which is shown in frame 102.
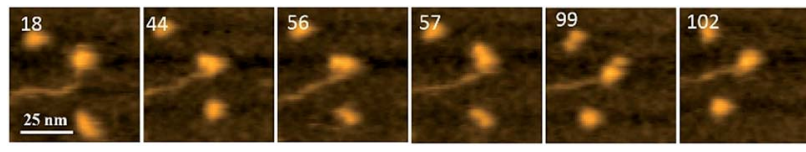

Fig. 1 AFM selected frames from Movie $1 \uparrow$ illustrating the dynamics of A3G in complex with the 69 nt tail DNA. Frames 18 and 102 show the globular structure of A3G in complex with ssDNA. Frames 44, 56, 57, and 99 represent the dumbbell structure of $A 3 G$ in complex with ssDNA. The average yield of dumbbell structures is $65 \%$. The scale bar is $25 \mathrm{~nm}$. The scan rate is $398 \mathrm{~ms}$ per frame.
The first striking observation for A3G in complex with the 69 nt tail ssDNA substrate was the high yield of the dumbbell structures. The average yield for the dumbbell structure was $65 \%$, analyzed from 10 separate movies with a total of $\sim 600$ frames. Note that this yield is four times greater than the yield of A3G dumbbells for A3G not bound to ssDNA.

For quantitative characterization of the dumbbell and globular structures of A3G in complex with the $69 \mathrm{nt}$ tail ssDNA, several parameters were used, as shown in Fig. 2. For the dumbbell structure of A3G, the cross-sectional feature was selected, as shown in Fig. 2A (marked with a red line on the AFM image). Fig. 2B illustrates three parameters, calculated from the cross-section of the dumbbell structure of A3G. The height of each maximum is marked as $h 1$ for Domain 1 and $h 2$ for Domain 2; the center-to-center distance is marked as $d$ between domains. For the globular structure of A3G, as shown in the AFM image in Fig. 2C, the ratio between two orthogonal diameters $d 1: d 2$ was used, marked as blue and red lines, respectively. The plot in Fig. 2D illustrates measurements for two cross-sections of the globular structure.

Fig. 3 shows results from data analysis for the dumbbell and globular structures of A3G. Fig. 3A shows the dependence of the distance $(d)$ between the two A3G domains on the frame number, calculated for the dumbbell structure of the A3G-69 nt tail ssDNA complexes. These data show a wide range of fluctuation in the distances between the two domains, between $3 \mathrm{~nm}$ and $8 \mathrm{~nm}$. Fig. 3B provides a histogram for the distribution of the distance $(d)$ between the two domains in the dumbbell structure of A3G, and the Gaussian fit gives the average distance of $d=5.1 \pm 1.0 \mathrm{~nm}$. Fig. 3C shows the result for globular A3G in the complex as a dependence of the $d 1: d 2$ ratio on the frame
A

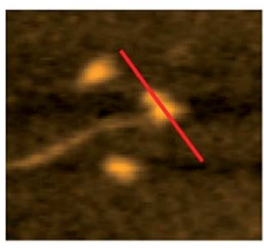

C

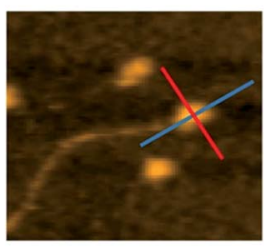

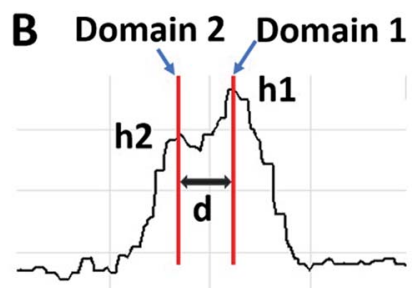

D

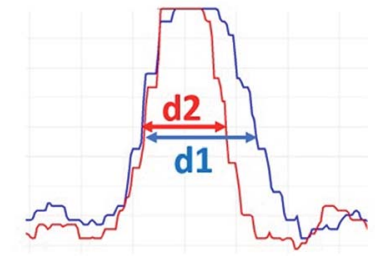

Fig. 2 Schematics explaining the analysis of various types of complexes of A3G with DNA. (A) AFM image of the A3G-69 nt ssDNA complex. The red line shows the cross-section of the dumbbell structure for the A3G-ssDNA complex. (B) Cross-sectional measurements of the heights of domains $(h 1)$ and $(h 2)$ and the distance $(d)$ between them. (C) AFM image of the globular structure of the A3G-69 nt ssDNA complex. Red and blue lines show the orthogonal crosssections of the A3G-ssDNA complex. (D) Cross-sectional measurements of the two orthogonal diameters, $d 1$ and $d 2$. 
A
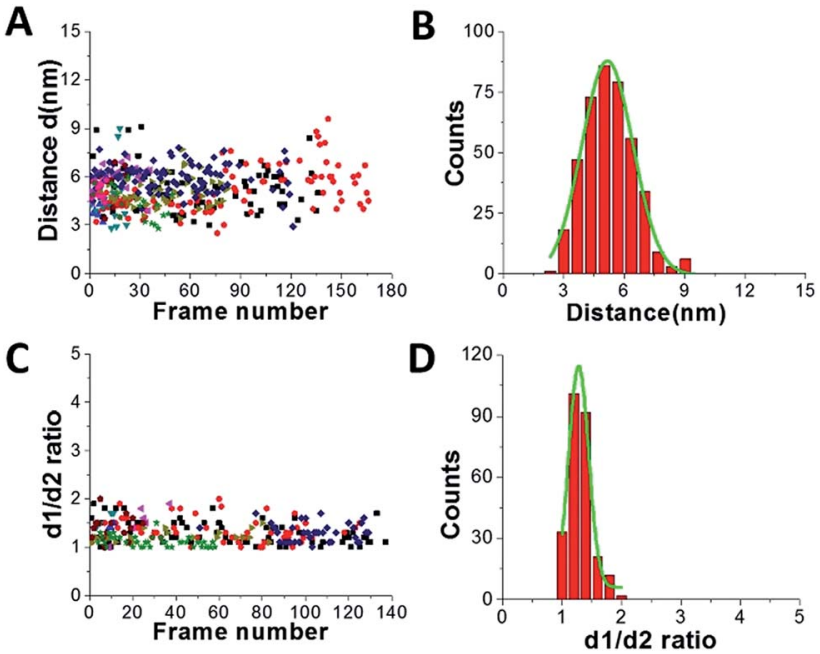

Fig. 3 Data analysis for the A3G-69 nt tail ssDNA complex. (A) The dependence of distance $(d)$ between Domain 1 and Domain 2 on the frame number for the A3G-69 nt tail ssDNA complex. (B) The histogram of distances between the two domains. The mean value for distances $(d)$ between Domain 1 and Domain 2 together with standard deviation is $5.1 \pm 1.0 \mathrm{~nm}$. (C) The dependence of the $d 1: d 2$ ratio for the globular structure of $A 3 G$ on frame number for the A3G-69 nt tail ssDNA complex. (D) The histogram for the $d 1: d 2$ ratio. The mean value for $d 1: d 2$, with the standard deviation, is $1.3 \pm 0.2$. The data are the result of analysis of $\sim 600$ frames from 10 separate movies (molecules). Each color corresponds to a different molecule.

number and as a $d 1: d 2$ histogram (Fig. 3D). The Gaussian fit to the histogram produces a mean value for the $d 1: d 2$ ratio of 1.3 \pm 0.2 , which resembles the data for free A3G.

Another important parameter, which can be obtained from the HS-AFM data, is the lifetime for the specific structure of A3G in the complex. Fig. 4A shows a plot for the dependence of the distance $(d)$ between the two domains in the dumbbell structure (right axes, blue) and $d 1: d 2$ for the globular structure (left, black) for the A3G-69 nt tail ssDNA complex on the frame number, obtained from one of the movies. Blue dots show changes in the distance $(d)$ between A3G domains in the dumbbell structure, and black triangles represent fluctuations in the $d 1: d 2$ ratio for the globular structure. Following frameby-frame transitions between globular and dumbbell structures, the lifetime was calculated for each structure of A3G in the complex. The zoomed portion of the plot in Fig. 4A (marked by a red rectangle) is shown in Fig. 4B, where several consecutive, uninterrupted frames for the dumbbells characterize their lifetime (blue dots), and likewise, several uninterrupted frames for the globular structure (black triangles) characterize the lifetimes of the globular structure. Fig. S2† offers another example of the dynamic behavior of the dumbbell structure of A3G in the DNA complex. The plot in Fig. S2† illustrates an example of the long-lived dumbbell structure of A3G in complex with the $69 \mathrm{nt}$ tail ssDNA substrate, with large fluctuations in the distance $(d)$ between the two domains. Analysis of the lifetimes obtained from all assembled movies for the A3G-69 nt tail ssDNA complexes is shown as histograms in Fig. 4 for the dumbbells (C) and globular (D) A3G structures. The fit of these
A

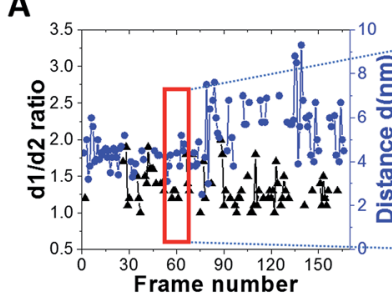

B
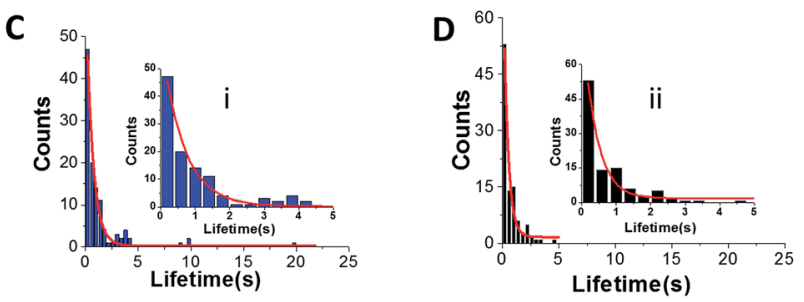

Fig. 4 Dynamics of A3G in complexes with 69 nt tail ssDNA. (A) Plot illustrating the dynamics of A3G in complex with the 69 nt tail ssDNA. The blue dots represent the distance $(d)$ between the two domains for the dumbbell structure of $A 3 G$. Black triangles represent the ratio of the two orthogonal diameters, $d 1: d 2$, for the globular structure of A3G. (B) Zoomed view of (A) (marked by a red rectangle) for the dumbbell (blue dots) and globular (black triangles) structures of A3G. The arrows show examples for calculation of the lifetime for dumbbell structures "i" (blue) and globular "ii" (black) for A3G. (C) The histogram for the lifetime of $A 3 G$ in the dumbbell structure, which after fitting with the first-order exponential model gives a lifetime of $\sim 0.64 \pm 0.03$ seconds. (D) The histogram for the lifetime of A3G in the globular structure, which after fitting with the first-order exponential model gives a lifetime of $\sim 0.39 \pm 0.06$ seconds. Insets are zoomed parts from frames $(C(i))$ and $(D(i i))$.

histograms with first-order exponential decay gives a lifetime of $0.64 \pm 0.03$ seconds for dumbbells and $0.39 \pm 0.06$ seconds for globular structures.

\section{A3G in complex with the 25 nt tail DNA substrate}

To understand the role of the length of ssDNA substrate plays in the structure and dynamics of A3G, the length of the ssDNA substrate was reduced to $25 \mathrm{nt}$. The selected frames from Movie $2, \dagger$ as shown in Fig. 5, demonstrate the structure and dynamics of monomeric A3G in complex with the $25 \mathrm{nt}$ tail ssDNA. In this complex, A3G also reveals both structures: globular (in frames 1 and 35) and dumbbell (in frames 19 and 38). However, the estimated yield of the dumbbells, calculated from 24 separate movies and $\sim 600$ frames in total, was $35 \%$, which is roughly two
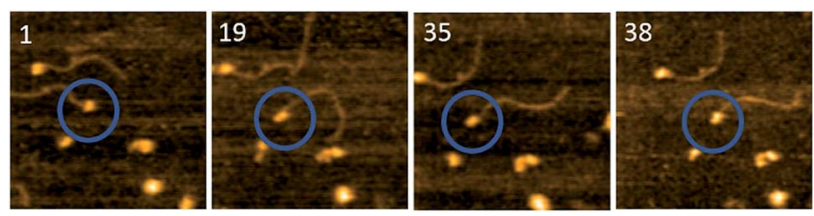

Fig. 5 Selected frames illustrating the dynamics of A3G in complex with the $25 \mathrm{nt}$ tail ssDNA. Circles show the complex of interest. Frames 1 and 35 represent the globular structure of $A 3 G$ and frames 19 and 38 show the dumbbell structure. The average yield of dumbbell structures is $35 \%$. The scan size is $200 \mathrm{~nm}$ and the scan rate is $398 \mathrm{~ms}$ per frame. 
times less than that of the 69 nt tail ssDNA substrate. Similarly to the analysis of the A3G-69 nt tail ssDNA complexes, data were analyzed for the A3G-25 nt tail ssDNA complexes and the results are presented in Fig. 6 . The dependence of the distance $(d)$ on the frame number illustrates the dynamic properties of the dumbbell structure of A3G, as shown in Fig. 6A. A histogram for the distance $(d)$ is shown in Fig. 6B. For the dumbbell structure of $\mathrm{A} 3 \mathrm{G}$, the mean distance is $d=4.7 \pm 1.0 \mathrm{~nm}$, which is slightly less than that for A3G in complex with the $69 \mathrm{nt}$ tail ssDNA, which is $5.1 \pm 1.0 \mathrm{~nm}$. The results for the globular structure show the dependence of $d 1: d 2$ on the frame number (Fig. 6C); the histogram for $d 1: d 2$ is shown in Fig. 6D. The calculated lifetimes for dumbbell and globular structures are shown in Fig. $6 \mathrm{E}$ and $\mathrm{F}$, which show that the lifetime of the dumbbell structure for A3G in complex with the $25 \mathrm{nt}$ tail ssDNA is less than that of the globular structure: $0.42 \pm 0.01$ seconds and 1.29 \pm 0.09 seconds, respectively.

\section{A3G in complex with the 69 nt gap DNA substrate}

The results for the $69 \mathrm{nt}$ tail ssDNA substrate show that the position of one of the domains in the dumbbell structure of A3G changes relative to the dsDNA tag (Fig. 1, frames 56 and 57). Additionally, one of the domains of A3G appears smaller in size.

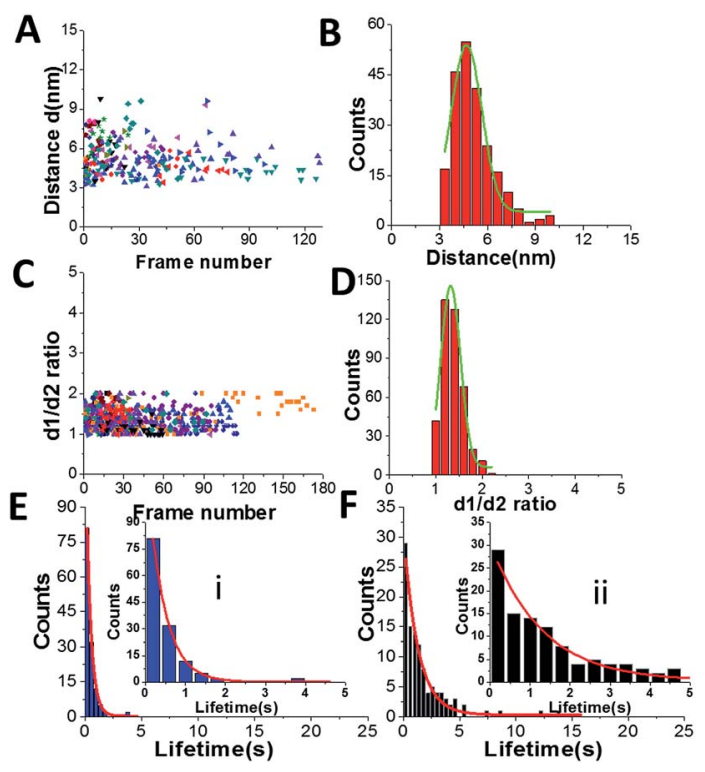

Fig. 6 Data analysis for the A3G-25 nt tail ssDNA complex. (A) The distance $(d)$ between Domain 1 and Domain 2 for the dumbbell structure of $A 3 G$ in the $A 3 G-25$ nt tail ssDNA complex. (B) The histogram of the distances between the two domains. The mean value for distances between Domain 1 and Domain 2 together with the standard deviation is $4.7 \pm 1.0 \mathrm{~nm}$. (C) The dependence of the $d 1: d 2$ ratio for the globular structure of $A 3 G$ in the $A 3 G-25$ nt tail ssDNA complex on the frame number. (D) The histogram for the $d 1: d 2$ ratio. The mean value for $d 1: d 2$, with the standard deviation, is $1.3 \pm 0.2$. (E) The lifetime of the dumbbell structure of A3G in complex with the 25 $\mathrm{nt}$ tail ssDNA. After fitting, the lifetime is $0.42 \pm 0.01 \mathrm{~s}$. (F) The lifetime of the globular structure of A3G in complex with 25 nt tail ssDNA. After fitting, the lifetime is $1.29 \pm 0.09 \mathrm{~s}$. The data are the results of analysis of 600 frames from 18 separate movies (molecules). Insets are zoomed parts from $(E(i))$ and $(F(i i))$.
This observation indicates a possible transient dissociation of one of the domains from the ssDNA substrate. To directly visualize and characterize a possible transient dissociation of one of the domains from the ssDNA substrate, the $69 \mathrm{nt}$ gap ssDNA substrate was used, where $69 \mathrm{nt}$ ssDNA was fused between two dsDNA duplexes (Fig. S1C $\dagger$ ).

Fig. 7 presents selected frames from Movie $3, \dagger$ where the transient dissociation of one of the A3G domains from the ssDNA substrate is unambiguously seen. Frames 21, 25, 47, and 56 show one smaller-sized domain unbound to the ssDNA substrate. Frames 175, 182, 187, and 196 show both domains, similar in size, bound to the ssDNA gap substrate. A3G also formed a globular, compact structure, as seen in frames 42 and 73.

The smaller size of such a domain can be explained by its lack of binding to the ssDNA substrate, which may contribute to the overall size of the domain. To confirm this effect, the ratios of the heights of Domain $1(h 1)$ to those of Domain $2(h 2)$ were calculated (Fig. 2B). Data for the $h 1: h 2$ ratio are incorporated into frames in Fig. 7. When both domains are in the dumbbell structure and bound to the substrate, DNA contributes equally to the sizes of the domains. Therefore, the ratio of heights of the domains $h 1: h 2$ would be expected to be close to one, which is clearly seen in frames $175,182,187$, and 196. Meanwhile, when one of the domains is unbound to the ssDNA substrate, the $h 1: h 2$ ratio should increase due to the lack of binding of this domain with the ssDNA substrate, as seen in frames $21,25,47$, and 56.

\section{Discussion}

The data presented demonstrate the structure and dynamics of full-length, monomeric A3G in complex with ssDNA substrates. The continuous, frame-by-frame HS-AFM imaging of A3GssDNA complexes allowed for clear visualization of not only the dumbbell and globular structures of A3G in complex with ssDNA substrates, but also the transition between them. The major finding here is the high yield of A3G dumbbell structures

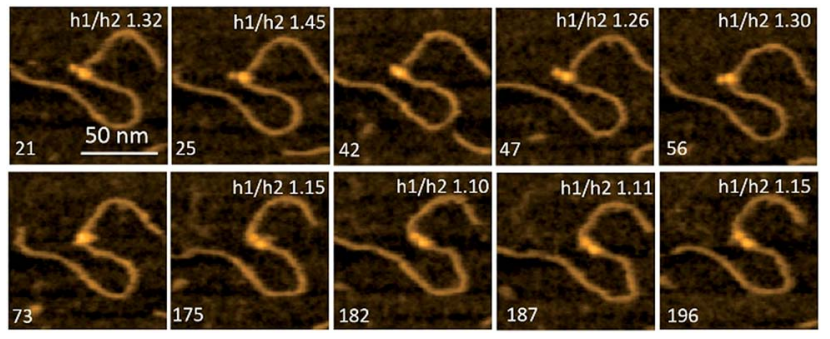

Fig. 7 Selected frames from Movie $3 \dagger$ illustrating the different positions of A3G domains in complex with the 69 nt gap DNA. Frames 21, 25,47 , and 56 show the dumbbell structure of $A 3 G$ with one domain unbound to the ssDNA substrate. Frames 42 and 73 represent the globular structure of A3G. Frames 175, 182, 187, and 196 represent the dumbbell structure of A3G with both domains located on the sSDNA substrate. The ratio of the height of Domain 1 to that of Domain 2 ( $h 1: h 2)$ of A3G is inserted at the top of each frame. The scale bar is $50 \mathrm{~nm}$. The scan rate is $398 \mathrm{~ms}$ per frame. 
in complex with ssDNA substrates compared to the protein nonbound to ssDNA, ${ }^{28}$ suggesting that the interaction with ssDNA substrates shifts the conformational equilibrium of $\mathrm{A} 3 \mathrm{G}$ to the dumbbell conformation.

The yield of the dumbbell conformation of A3G also depends on the length of the ssDNA substrate. Table 1 summarizes data obtained from analyses of the dumbbell and globular structures of A3G in complex with 69 nt and 25 nt tail ssDNA substrates and free A3G. As seen in Table 1, in the presence of a long $69 \mathrm{nt}$ ssDNA substrate, the dumbbell structure shows the highest yield of dumbbells (65\%), which drops to 35\% for a shorter, 25 nt ssDNA substrate, and comprises only $16 \%$ of free A3G. Together these data clearly demonstrate the effect of the ssDNA substrate on conformational changes of A3G domains and show the dependence of such changes on the length of the ssDNA substrate. The average distance between A3G domains for the dumbbell structures in A3G-SsDNA complexes tends to change slightly, from $5.1 \pm 1.0 \mathrm{~nm}$ for a long substrate and decreasing up to $4.7 \pm 1.0 \mathrm{~nm}$ for a shorter one, the smallest being $4.4 \pm$ $0.9 \mathrm{~nm}$ for free A3G. Data for the globular structure do not demonstrate changes for A3G ssDNA complexes and free A3G, indicating that the ssDNA substrate does not affect the globular structure of $\mathrm{A} 3 \mathrm{G}$. Indeed, the $d 1: d 2$ ratio remains equal to 1.3, indicating the elongated shape for both A3G in complex with ssDNA and free A3G.

HS-AFM data also reveal a different affinity for the A3G domains in the dumbbell structure to the DNA substrate. As seen in Fig. 7, one of the A3G domains in complex with ssDNA is capable transiently dissociating from the ssDNA substrate. Quantitatively, for the dumbbell structure of A3G in the complex, this effect is illustrated by measuring of ratios of the heights of Domain 1 to those of Domain $2(h 1: h 2)$. The value of the $h 1: h 2$ ratio is close to one when both domains are bound to the substrate, but when one of the domains is unbound to the ssDNA the $h 1: h 2$ ratio is 1.3 . These measurements were performed for ssDNA substrates with both the $69 \mathrm{nt}$ and $25 \mathrm{nt}$ tail ssDNA substrates. Fig. $8 \mathrm{~A}$ and $\mathrm{B}$ present the results of this analysis. Histograms for A3G complexes with $69 \mathrm{nt}$ and $25 \mathrm{nt}$ tail ssDNA substrates have two distinct peaks. The first peak, with almost equal heights of the domains, corresponds to cases when both domains are bound to the substrate. The second peak corresponds to cases when one of the domains is unbound to the substrate, with the $h 1: h 2$ ratio close to 1.3 , indicating the contribution of ssDNA to the size of the domain. Comparatively, for free A3G (Fig. S3†), the histogram shows only one maximum for the ratio $h 1: h 2$, which is close to one. Another line of evidence for the contribution of ssDNA to the overall size of the A3G domains comes from directly measuring the heights of each domain for free A3G and A3G in complex with 69 nt tail
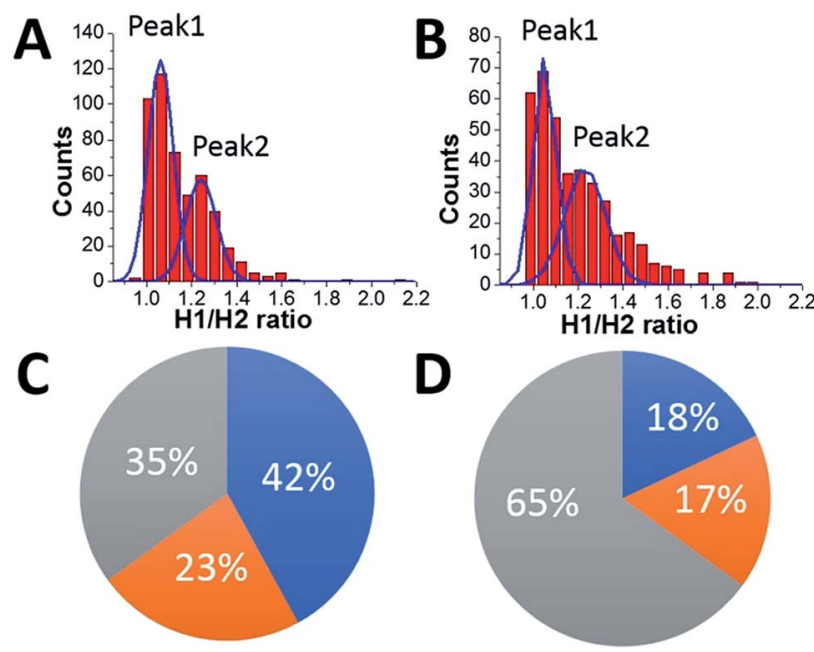

Fig. 8 The ratio of the heights of Domain 1 to those of Domain 2 ( $h 1$ : h2). (A) The A3G-69 nt tail ssDNA complex. (B) The A3G-25 nt tail ssDNA complex. The ratios of the areas under the first peak and the second peak are 1.8 for the A3G-69 nt ssDNA complex (A) and 1.1 for the A3G-25 nt ssDNA complex (B). The diagram represents the distribution of globular and dumbbell A3G structures for the $69 \mathrm{nt}$ tail ssDNA substrate (C) and the $25 \mathrm{nt}$ tail ssDNA substrate (D). The grey area shows the yield of globular A3G structures for the $69 \mathrm{nt}$ tail ssDNA substrate with $35 \%$ (C) and $65 \%$ for the 25 nt tail ssDNA substrate (D). The blue area illustrates both $A 3 G$ domains bound to the substrate, and the orange area shows one of the domains unbound to the substrate. For long substrates (C), both domains are bound to the substrate in $42 \%$ of cases and one domain is unbound from the substrate in $23 \%$ of cases. For a short substrate (D), both domains are bound to the substrate in $18 \%$ of cases, and one domain is unbound to the substrate in $17 \%$ of cases.

ssDNA, as shown in Fig. S4. $\dagger$ Here, we assembled histograms for the heights of each domain in the dumbbell structure for free A3G (Fig. S4A and $\mathrm{B} \dagger$ ) and for A3G in complex with the 69 nt tail SsDNA (Fig. S4C and $\mathrm{D}^{\dagger}$ ). Data demonstrate that the heights of the domains for free A3G are similar when compared to the heights of domains for A3G in the complex (Fig. S4C and $\mathrm{D}^{\dagger}$ ). Note that the height of one of the domains for $\mathrm{A} 3 \mathrm{G}$ in the complex with the ssDNA substrate is close to the height of both domains for free A3G (Fig. S4D †), which indicates that this domain is unbound to the ssDNA substrate (Fig. S4C $\dagger$ ). Overall, the data presented here clearly demonstrate that one of the domains in the dumbbell structure of A3G is capable of transiently dissociating from the ssDNA substrate, supported by the lack of the contribution of ssDNA substrate to the size of the protein.

The diagrams in Fig. 8C and D summarize the analysis of all the results obtained here. The grey area in the diagram presents

Table 1 The yield and the distance between domains in the dumbbell structure for free A3G and A3G in complex with ssDNA

\begin{tabular}{llll}
\hline & $69 \mathrm{nt}$ tail DNA-A3G & $25 \mathrm{nt}$ tail DNA-A3G & Free A3G \\
\hline Globular $d 1 / d 2$ & $1.3 \pm 0.2$ & $1.3 \pm 0.2$ & $1.3 \pm 0.3$ \\
Dumbbell yield & $65 \%$ & $35 \%$ & $16 \%$ \\
Dumbbell distance & $5.1 \pm 1.0 \mathrm{~nm}$ & $4.7 \pm 1.0 \mathrm{~nm}$ & $4.4 \pm 0.9 \mathrm{~nm}$
\end{tabular}


the yield of globular A3G structures, calculated to be $35 \%$ for the $69 \mathrm{nt}$ tail ssDNA substrate (A) and 65\% for the $25 \mathrm{nt}$ tail ssDNA substrate (B). The estimated lifetime for the globular A3G structure in complex with the $69 \mathrm{nt}$ tail ssDNA $(\sim 0.39 \pm 0.06 \mathrm{~s})$ tends to be less than that with the 25 nt tail ssDNA substrate $(\sim 1.29 \pm 0.09 \mathrm{~s})$. The shorter lifetime for the globular structure correlates with the reduced yield of the globular structure compared to the dumbbell structure for the A3G-69 nt ssDNA complexes. The blue and orange areas together show the yield of dumbbell structures for long and short ssDNA substrates to be $65 \%$ and $35 \%$, respectively, with a tendency toward increased lifetimes for the dumbbell structures in complex with $69 \mathrm{nt}$ ssDNA $(\sim 0.64 \pm 0.03 \mathrm{~s})$ compared with a shorter ssDNA substrate $(0.42 \pm 0.01 \mathrm{~s})$. These results show the correlation between the yield of dumbbell and globular structures of A3G and their lifetime on the different ssDNA substrates.

As shown in Fig. 8A and B, the two distinct peaks for $h 1: h 2$ values, shown for the long and short ssDNA substrates, demonstrate different positions of A3G domains on the ssDNA substrate. Indeed, when both domains are bound to the SsDNA substrate, the $h 1: h 2$ ratio is close to one, compared to the $h 1: h 2$ ratio equal to 1.3 when one of the domains is unbound to the substrate. In addition to the position of the domains in dumbbell structures of A3G in A3G-ssDNA complexes discussed above, the areas under peak 1 and peak 2 (Fig. 8A and B) indicate the different number of events for bound and unbound domains for long and short ssDNA substrates. Indeed, for a long substrate, the ratio between areas under peak 1 and peak 2 is 1.8 , indicating an almost twice greater number of events when both A3G domains are positioned on the ssDNA compared to one of the domains being unbound. The blue and orange areas in Fig. 8C show such a distribution to be $42 \%$ for both domains bound to the ssDNA substrate (blue area) vs. $23 \%$ for the unbound one (orange area). For a short ssDNA substrate (Fig. 8D), the ratio between areas under peak 1 and peak 2 is 1.1 , demonstrating a practically equal number of events for A3G domains positioned on the substrate and for one domain unbound, as shown in blue (18\%) and orange (17\%) areas in the diagram, respectively.

HS-AFM is not capable of identifying which domain remains in contact with the ssDNA and which is temporarily dissociated. Nevertheless, several lines of evidence allow us to posit that the CTD is the domain capable of transiently dissociating from the ssDNA. Computer analysis performed ${ }^{35}$ shows that the isoelectric point (pI) of the N-terminal domain (NTD) is 9.6, compared to 6.9 for the CTD. In addition, the number of aromatic amino acids in A3G essential for ssDNA binding is 9 for the NTD versus only 6 for the CTD. Taken together, these findings suggest tighter binding for the NTD than for the CTD. Also, more stable binding of the NTD with SSDNA than of the CTD has been reported. ${ }^{28,35,36}$ Moreover, it is demonstrated that the NTD is responsible not only for binding with SSDNA,,$^{35,36}$ but also for positioning and stabilizing active sites of the CTD for efficient deamination of ssDNA. ${ }^{37}$ Mutational studies $^{38}$ suggest the following two steps for A3G binding with the ssDNA template: (1) initially, high affinity binding is carried out by the NTD with $K_{\mathrm{d}}$ in the nM range, (2) followed by the CTD with $K_{\mathrm{d}}$ in the $\mu \mathrm{M}$ range. In addition, the data obtained in ref. 39 and 40 have demonstrated that during A3G sliding, the CTD tends to dissociate from ssDNA. Therefore, we hypothesize that the CTD has greater conformational mobility compared to the NTD, and is capable of transiently dissociating from the ssDNA template.

Based on our data, we suggest a model where the substrate length is key in determining whether a dumbbell or globular structure will form on each ssDNA substrate. Fig. 9 illustrates such a model for long (A) and short (B) ssDNA substrates. The red ball represents the CTD, which forms a dumbbell structure and is unbound to the SsDNA, and the blue ball represents the NTD bound to ssDNA (state i). In this state (i), only the NTD is bound to the substrate, and A3G may dissociate from a long or short substrate with equal probability. This would explain the similar number of cases when only one domain is bound to the substrate for both long and short ssDNA substrates, $23 \% v s$. $17 \%$, respectively (Fig. 8C and D, orange area). If not dissociated, as in the case of a long substrate (A), the CTD may return to the substrate and preserve the dumbbell structure (grey arrows, state ii) with both domains bound to ssDNA; alternatively, A3G may come close to the NTD domain to form a globular structure (purple arrow, state iii). In the case of a long substrate, A3G has a greater chance of holding the dumbbell structure with both domains bound to the ssDNA, as shown in Fig. 8C (blue area). Therefore, it is reasonable to theorize that for a long substrate, the increased yield of dumbbell structures is primarily due to both domains being bound to the substrate. However, this differs for a short substrate (B). Indeed, the CTD in state i may return to the NTD to form a globular shape (purple arrow, state iii) or form a preserved dumbbell structure with both domains bound to the substrate (grey arrow, state ii) or one domain dissociated from the substrate (orange arrow, state iv). However, for a short substrate, there is less possibility to preserve the dumbbell structure with two domains bound to the substrate, which comprises $18 \%$ (Fig. 8D, blue area), compared to $42 \%$ for a long substrate (Fig. $8 \mathrm{C}$, blue area).

The conformational changes between domains, facilitated by an interdomain linker, ${ }^{28}$ are more easily achieved when A3G adopts a dumbbell structure and may facilitate functions of A3G such as sliding ${ }^{\mathbf{8 1 9 , 4 1}}$ and intersegmental transfer ${ }^{\mathbf{1 6}}$ and
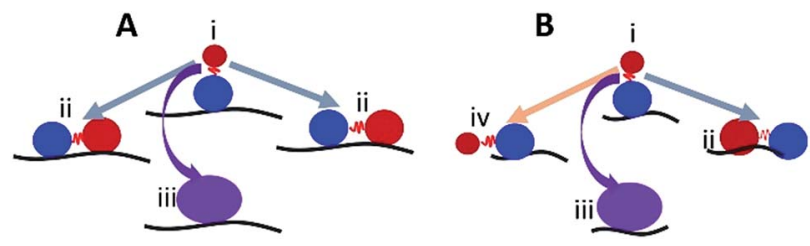

Fig. 9 The model explaining the role of the dumbbell conformation of A3G in the assembly complexes with long (A) and short (B) ssDNA. The red and blue balls represent the CTD and NTD, respectively. State i illustrates the dumbbell structure with one $A 3 G$ domain unbound to the ssDNA substrate. In state i, A3G is capable of transiently dissociating from/associating with the substrate. In state ii, both domains are bound to the ssDNA substrate (grey arrows) or form a globular structure (iii) bound to the ssDNA substrate (purple arrow). State iv shows one domain unbound in the case of a short substrate (orange arrow). 
eventually the search for the deamination target of the ssDNA substrate. Our data demonstrate that one of the domains is capable of transiently dissociating from the substrate, and such dynamics may facilitate the search for the deamination target. Moreover, we suggest that the CTD is the domain that transiently dissociates from the substrate to facilitate this search. Based on our data, we posit that the dumbbell structure of A3G represents an active structure of the protein. Interestingly, a decrease in the yield for dumbbell structures with a short substrate correlates with the length dependence of deaminase activity of A3G. ${ }^{\mathbf{8} 42,43}$ Indeed, it was shown ${ }^{\mathbf{8}}$ that specific activity of A3G increases between $15 \mathrm{nt}$ and $60 \mathrm{nt}$ ssDNA lengths and remains unchanged thereafter. Despite the fact that both globular and dumbbell forms of A3G provide efficient binding with the ssDNA substrate, a correlation between lengthdependence of deaminase activity and the yield of dumbbells supports our hypothesis that dumbbell structures of A3G represent an active form of the protein. Given that $A 3 G$ is dynamic and in the extended dumbbell conformation occupies a space as long as $\sim 10 \mathrm{~nm}$, this property of A3G is a factor that defines the interdomain dynamics of the protein. Indeed, $10 \mathrm{~nm}$ corresponds to an ssDNA length of $\sim 30 \mathrm{nt}$, and we did observe the decrease of dumbbell conformation for the $25 \mathrm{nt}$ ssDNA substrate.

\section{Materials and methods}

\section{Hybrid ssDNA substrates}

The 69 nt tail ssDNA. The hybrid 69 nt tail ssDNA was assembled as previously described. ${ }^{33}$ Briefly, the synthesized (Integrated DNA Technology; IA) 89 nt oligo was annealed at a $1: 1$ ratio with a phosphorylated 23 nt oligo (Integrated DNA Technology, IA) to form a 20 bp DNA duplex with sticky ends. Later, the construct was ligated at $16{ }^{\circ} \mathrm{C}$ overnight with a previously gel-purified 356 bp DNA fragment with sticky ends. The ligated product was purified from the gel using a QIAquick Gel Extraction Kit (Qiagen) as described ${ }^{33}$ and re-suspended in TE buffer containing $10 \mathrm{mM}$ Tris, $\mathrm{pH}$ 7.5, and $1 \mathrm{mM}$ EDTA. The final product consists of the $69 \mathrm{nt}$ ssDNA attached to a $379 \mathrm{bp}$ dsDNA fragment as a tag.

The 25 nt tail ssDNA. The hybrid 25 nt tail ssDNA was assembled according to the procedure described above for the 69 nt tail ssDNA. In this case, synthesized 58 nt oligos (Integrated DNA Technology, IA) were annealed with phosphorylated $20 \mathrm{nt}$ oligos to create a $33 \mathrm{bp}$ duplex with a sticky end to ligate with a 224 bp DNA fragment. The final product consists of the $25 \mathrm{nt}$ tail ssDNA attached to a $260 \mathrm{bp}$ dsDNA as a tag.

The 69 nt gap ssDNA. Creation of the hybrid DNA substrate, in which an ssDNA region is flanked by dsDNA arms, has been previously described in detail. ${ }^{\mathbf{4 4 , 4 5}}$ First, 235 bp dsDNA and 441 bp dsDNA fragments with sticky ends were generated by PCR and purified from the gel. Second, $235 \mathrm{bp}$ hybrid $5^{\prime}$ end tail ssDNA and 441 bp hybrid $3^{\prime}$ end tail ssDNA substrates were prepared as described above for preparation of hybrid tail ssDNA substrates. Third, two hybrid $3^{\prime}$ and $5^{\prime}$ end tail ssDNA substrates were mixed in a $1: 1$ ratio and annealed with the bridge oligo. Next, the annealed product was ligated at $16{ }^{\circ} \mathrm{C}$ overnight. To remove the bridge oligo, the product was heated to $70{ }^{\circ} \mathrm{C}$ for 5 minutes and immediately put into ice. Finally, the 69 nt gap DNA substrate was gel purified using a QIAquick Gel Extraction Kit (Qiagen), as described. ${ }^{33}$ The final product consists of 69 nt ssDNA flanked with 441 bp and 235 bp dsDNA, respectively.

\section{Preparation of A3G in complex with ssDNA substrates}

For each ssDNA substrate mentioned above, a complex with A3G was formed in a $4: 1$ protein-to-ssDNA ratio in binding buffer containing $50 \mathrm{mM}$ HEPES, pH 7.5, $100 \mathrm{mM} \mathrm{NaCl}, 5 \mathrm{mM}$ $\mathrm{MgCl}_{2}$, and $1 \mathrm{mM}$ DTT. The complex was incubated for 15 minutes at $37^{\circ} \mathrm{C}$ before deposition on a mica surface. Fig. $\mathrm{S} 1 \dagger$ schematically shows the positions of A3G on different ssDNA substrates.

\section{Sample preparation for HS-AFM}

A detailed description of the sample preparation for HS-AFM has been previously described. ${ }^{17}$ In brief, a small piece of mica, glued to a cylinder, was cleaved and treated with APS, as described. ${ }^{17}$ Two microliters of the complexes were deposited on the APS mica surface for 2 minutes, followed by washing with binding buffer. Continuous scanning was initiated immediately following the wash, without drying of the sample. The selected scanning area $(200 \mathrm{~nm} \times 200 \mathrm{~nm})$ was continuously imaged to visualize the dynamics of the complexes at a scan rate of $398 \mathrm{~ms}$ per frame. The tips for imaging were grown under an electron beam using short cantilevers (BL-AC10DS-A2, Olympus; Tokyo, Japan) with a spring constant between 0.1 and $0.2 \mathrm{~N} \mathrm{~m}^{-1}$ and a resonance frequency of $400-1000 \mathrm{kHz}$.

\section{Analysis of the HS-AFM data}

After collecting frame-by-frame HS-AFM images for A3G in complex with different ssDNA substrates, a set of movies was assembled. Analysis of these movies revealed the following two structures for A3G in the complexes: dumbbell and globular. To analyze the data obtained from HS-AFM experiments, the crosssectional feature was used in FemtoScan Online software (Advance Technologies Center; Moscow, Russia), as previously described. ${ }^{28,33,46}$ Analysis was completed for each frame from the collected movies. More than 500 frames were analyzed for each A3G structure in the A3G-SsDNA complexes.

\section{Conclusions}

In summary, the data presented here support the important role of an ssDNA substrate in the dynamics of A3G, demonstrating different distributions between globular and dumbbell structures of A3G in the complex. The results show not only a higher yield of the dumbbell structures of A3G in the A3G-SsDNA complex compared to free $\mathrm{A} 3 \mathrm{G}$, but also the dependence of the yield of dumbbells on the ssDNA length. Our results also identified different binding affinity of the A3G domain to the ssDNA substrate. 


\section{Conflicts of interest}

There are no conflicts to declare.

\section{Acknowledgements}

This work was supported by NIH grants awarded to Dr Lyubchenko (R01-GM118006, GM096039, and R21NS101504). We thank Dr R. Harris for providing A3G protein and Dr Lyubchenko's lab members for their fruitful discussions. We thank Mohtadin Hashemi for his productive and helpful discussions regarding this work and Dr Atsushi Miyagi for the HS AFM movie of the $69 \mathrm{nt}$ gap ssDNA-A3G complex. Lastly, we thank Melody A. Montgomery for the professional editing of this manuscript.

\section{References}

1 R. S. Harris, K. N. Bishop, A. M. Sheehy, H. M. Craig, S. K. Petersen-Mahrt, I. N. Watt, M. S. Neuberger and M. H. Malim, Cell, 2003, 113, 803-809.

2 J. D. Salter, J. Krucinska, J. Raina, H. C. Smith and J. E. Wedekind, Biochemistry, 2009, 48, 10685-10687.

3 J. D. Salter, R. P. Bennett and H. C. Smith, Trends Biochem. Sci., 2016, 41(7), 578.

4 R. S. Harris and J. P. Dudley, Virology, 2015, 479-480, 131145.

5 A. Ara, R. P. Love, T. B. Follack, K. A. Ahmed, M. B. Adolph and L. Chelico, J. Virol., 2017, 91.

6 H. C. Smith, R. P. Bennett, A. Kizilyer, W. M. McDougall and K. M. Prohaska, Semin. Cell Dev. Biol., 2012, 23, 258-268.

7 L. Chelico, P. Pham and M. F. Goodman, Nat. Struct. Mol. Biol., 2009, 16, 454-455; author reply 455-456.

8 L. Chelico, P. Pham, P. Calabrese and M. F. Goodman, Nat. Struct. Mol. Biol., 2006, 13, 392-399.

9 Y. Feng, T. T. Baig, R. P. Love and L. Chelico, Front. Microbiol., 2014, 5, 450.

10 Y. Iwatani, D. S. Chan, F. Wang, K. S. Maynard, W. Sugiura, A. M. Gronenborn, I. Rouzina, M. C. Williams, K. MusierForsyth and J. G. Levin, Nucleic Acids Res., 2007, 35, 70967108.

11 K. R. Chaurasiya, M. J. McCauley, W. Wang, D. F. Qualley, T. Wu, S. Kitamura, H. Geertsema, D. S. Chan, A. Hertz, Y. Iwatani, J. G. Levin, K. Musier-Forsyth, I. Rouzina and M. C. Williams, Nat. Chem., 2014, 6, 28-33.

12 A. Okada and Y. Iwatani, Front. Microbiol., 2016, 7, 2027.

13 K. Belanger, M. Savoie, M. C. Rosales Gerpe, J. F. Couture and M. A. Langlois, Nucleic Acids Res., 2013, 41, 7438-7452.

14 F. Navarro, B. Bollman, H. Chen, R. Konig, Q. Yu, K. Chiles and N. R. Landau, Virology, 2005, 333, 374-386.

15 K. Shindo, A. Takaori-Kondo, M. Kobayashi, A. Abudu, K. Fukunaga and T. Uchiyama, J. Biol. Chem., 2003, 278, 44412-44416.

16 R. Nowarski, E. Britan-Rosich, T. Shiloach and M. Kotler, Nat. Struct. Mol. Biol., 2008, 15, 1059-1066.
17 L. S. Shlyakhtenko, A. Y. Lushnikov, A. Miyagi, M. Li, R. S. Harris and Y. L. Lyubchenko, J. Struct. Biol., 2013, 184, 217-225.

18 B. Polevoda, W. M. McDougall, R. P. Bennett, J. D. Salter and H. C. Smith, Methods, 2016, 107, 10-22.

19 L. Chelico, E. J. Sacho, D. A. Erie and M. F. Goodman, J. Biol. Chem., 2008, 283, 13780-13791.

20 Y. Feng and L. Chelico, J. Biol. Chem., 2011, 286(13), 11415. 21 X. Xiao, S. X. Li, H. Yang and X. S. Chen, Nat. Commun., 2016, $7,12193$.

22 E. Harjes, P. J. Gross, K. M. Chen, Y. Lu, K. Shindo, R. Nowarski, J. D. Gross, M. Kotler, R. S. Harris and H. Matsuo, J. Mol. Biol., 2009, 389, 819-832.

23 S. M. Shandilya, M. N. Nalam, E. A. Nalivaika, P. J. Gross, J. C. Valesano, K. Shindo, M. Li, M. Munson, W. E. Royer, E. Harjes, T. Kono, H. Matsuo, R. S. Harris, M. Somasundaran and C. A. Schiffer, Structure, 2010, 18, 28-38.

24 K. M. Chen, E. Harjes, P. J. Gross, A. Fahmy, Y. Lu, K. Shindo, R. S. Harris and H. Matsuo, Nature, 2008, 452, 116-119.

25 T. Kouno, E. M. Luengas, M. Shigematsu, S. M. Shandilya, J. Zhang, L. Chen, M. Hara, C. A. Schiffer, R. S. Harris and H. Matsuo, Nat. Struct. Mol. Biol., 2015, 22, 485-491.

26 X. Lu, T. Zhang, Z. Xu, S. Liu, B. Zhao, W. Lan, C. Wang, J. Ding and C. Cao, J. Biol. Chem., 2015, 290, 4010-4021.

27 A. Maiti, W. Myint, T. Kanai, K. Delviks-Frankenberry, C. Sierra Rodriguez, V. K. Pathak, C. A. Schiffer and H. Matsuo, Nat. Commun., 2018, 9, 2460.

28 S. Gorle, Y. Pan, Z. Sun, L. S. Shlyakhtenko, R. S. Harris, Y. L. Lyubchenko and L. Vukovic, ACS Cent. Sci., 2017, 3, 1180-1188.

29 T. Ando, T. Uchihashi and S. Scheuring, Chem. Rev., 2014, 114, 3120-3188.

30 N. Kodera, D. Yamamoto, R. Ishikawa and T. Ando, Nature, 2010, 468, 72-76.

31 T. Uchihashi, R. Iino, T. Ando and H. Noji, Science, 2011, 333, 755-758.

32 A. P. Nievergelt, N. Banterle, S. H. Andany, P. Gönczy and G. E. Fantner, Nat. Nanotechnol., 2018, 13, 696-701.

33 L. S. Shlyakhtenko, A. Y. Lushnikov, M. Li, L. Lackey, R. S. Harris and Y. L. Lyubchenko, J. Biol. Chem., 2011, 286, 3387-3395.

34 Y. Pan, K. Zagorski, L. S. Shlyakhtenko and Y. L. Lyubchenko, Sci. Rep., 2018, 8, 17953.

35 Y. Iwatani, H. Takeuchi, K. Strebel and J. G. Levin, J. Virol., 2006, 80, 5992-6002.

36 L. Chelico, C. Prochnow, D. A. Erie, X. S. Chen and M. F. Goodman, J. Biol. Chem., 2010, 285, 16195-16205.

37 K. Belanger and M. A. Langlois, Virology, 2015, 483, 141-148. 38 K. Shindo, M. Li, P. J. Gross, W. L. Brown, E. Harjes, Y. Lu, H. Matsuo and R. S. Harris, Biology, 2012, 1, 260-276.

39 K. Kamba, T. Nagata and M. Katahira, Front. Microbiol., 2016, 7, 587.

40 K. Kamba, T. Nagata and M. Katahira, Phys. Chem. Chem. Phys., 2016, 28(7), 587.

41 A. Ara, R. P. Love and L. Chelico, PLoS Pathog., 2014, 10, e1004024. 
42 S. Harjes, W. C. Solomon, M. Li, K. M. Chen, E. Harjes, R. S. Harris and H. Matsuo, J. Virol., 2013, 87, 7008-7014.

43 M. Mitra, K. Hercik, I. J. Byeon, J. Ahn, S. Hill, K. HincheeRodriguez, D. Singer, C. H. Byeon, L. M. Charlton, G. Nam, G. Heidecker, A. M. Gronenborn and J. G. Levin, Nucleic Acids Res., 2014, 42, 1095-1110.

44 L. S. Shlyakhtenko, A. Y. Lushnikov, A. Miyagi and Y. L. Lyubchenko, Biochemistry, 2012, 51, 1500-1509.
45 L. S. Shlyakhtenko, A. Y. Lushnikov, A. Miyagi, M. Li, R. S. Harris and Y. L. Lyubchenko, Biochemistry, 2012, 51, 6432-6440.

46 L. S. Shlyakhtenko, J. Gilmore, A. N. Kriatchko, S. Kumar, P. C. Swanson and Y. L. Lyubchenko, J. Biol. Chem., 2009, 284, 20956-20965. 